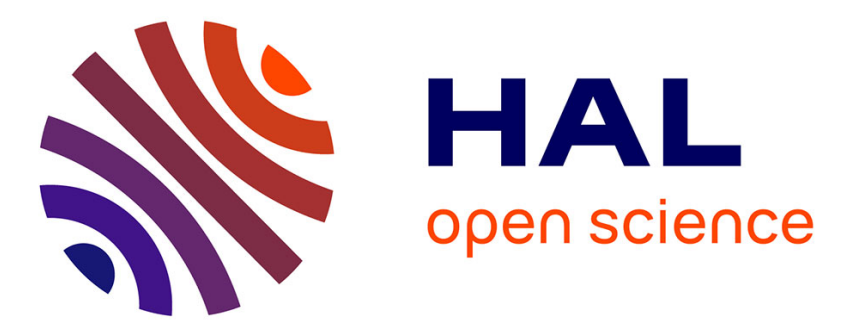

\title{
Prise en charge officinale des maux bénins durant la grossesse
}

\author{
Nicolas Clere
}

\section{To cite this version:}

Nicolas Clere. Prise en charge officinale des maux bénins durant la grossesse. Actualités Pharmaceutiques, 2019, 58, pp.41 - 43. 10.1016/j.actpha.2018.12.010 . hal-03486844

\section{HAL Id: hal-03486844 https://hal.science/hal-03486844}

Submitted on 20 Dec 2021

HAL is a multi-disciplinary open access archive for the deposit and dissemination of scientific research documents, whether they are published or not. The documents may come from teaching and research institutions in France or abroad, or from public or private research centers.
L'archive ouverte pluridisciplinaire HAL, est destinée au dépôt et à la diffusion de documents scientifiques de niveau recherche, publiés ou non, émanant des établissements d'enseignement et de recherche français ou étrangers, des laboratoires publics ou privés.

\section{다)(1) $(5$}

Distributed under a Creative Commons Attribution - NonCommerciall 4.0 International 
Version of Record: https://www.sciencedirect.com/science/article/pii/S0515370018304919

Manuscript_1b7d207606f8dde252812b7a2c1d7c9b

Dochead pratique

Sous-dochead conseil

\section{Prise en charge officinale des maux durant la grossesse}

\section{Nicolas Clere}

Maître de conférences

Faculté de santé, Département pharmacie, Université d'Angers, 16 boulevard Daviers, 49045 Angers, France

Adresse e-mail : nicolas.clere@univ-angers.fr (N. Clere).

Résumé

La femme enceinte est souvent confrontée à des maux bénins, certains dus à son statut physiologique, comme les troubles digestifs, d'autres n'ayant pas de lien avec son état, tels le rhume ou la toux. Dans ce contexte, la délivrance des médicaments fait appel à une extrême vigilance. Des alternatives aux médecines conventionnelles peuvent être proposées.

(C) 2018

Mots clés - grossesse ; pathologie ORL ; trouble digestif ; ; trouble du sommeil ; trouble veineux Summary à venir

(C) 2018

Keywords à venir

Les petits maux de la grossesse, bien que bénins, peuvent entraîner de véritables désagréments susceptibles de compromettre la qualité de vie de la femme enceinte, d'autant plus que les traitements qui peuvent lui être proposés sont peu nombreux.

\section{T1 Les troubles digestifs}

(C) 2018 published by Elsevier. This manuscript is made available under the CC BY NC user license https://creativecommons.org/licenses/by-nc/4.0/ 
Les troubles digestifs sont favorisés par les changements physiologiques inhérents à la grossesse. Leur prise en charge repose en première intention sur des conseils hygiéno-diététiques.

\section{T1 Le reflux gastro-œsophagien}

TEG1 Fréquent durant le troisième trimestre de la grossesse, le reflux gastro-œsophagien (RGO) est dû à l'hypotonie du sphincter inférieur de l'œsophage qui touche 50 à $80 \%$ des femmes enceintes [1]. Cette hypotonie est responsable de brûlures avec régurgitation acide ou amère, d'un pyrosis, fréquemment décrit en décubitus dorsal, et d'une œsophagite caractérisée par une brûlure lors de l'ingestion d'aliments chauds, sucrés et acides. Le diagnostic se base en général sur les signes cliniques [2].

TEG1 Avant de proposer une solution médicamenteuse, il convient de recommander à la patiente de supprimer ou diminuer la consommation des aliments irritants (alcool, épices...) et de ceux qui réduisent la pression du sphincter inférieur (chocolat, café...). Les repas peu abondants et fractionnés doivent être privilégiés. II faut également éviter de se coucher juste après avoir mangé.

TEG1 La prise en charge du RGO vise à neutraliser les sécrétions acides via l'utilisation, à distance des repas et au coucher, d'un anti-acide (Maalox ${ }^{\circledR}$, Phosphalugel ${ }^{\circledR}$ ). Devant une gêne importante, les alginates doivent être privilégiés (Topaal ${ }^{\circledR}$, Gaviscon $^{\circledR}$ ). Enfin, en cas d'échec, la prise de certains antiémétiques, disponibles sur prescription médicale, est envisageable.

TEG1 Des traitements homéopathiques peuvent être conseillés : Aesculus $5 \mathrm{CH}$ ou $7 \mathrm{CH}$ en cas de gastralgies brûlantes et d'éructations acides ; Argentum nitricum $5 \mathrm{CH}$ ou $7 \mathrm{CH}$ chez une patiente anxieuse présentant des gastralgies post-prandiales et des éructations bruyantes.

\section{T1 Les nausées et les vomissements}

TEG1 Plus de $\mathbf{8 0} \%$ des femmes enceintes souffrent de nausées ou de vomissements [3], sans que leur origine soit clairement identifiée [1]. Elles surviennent le plus souvent aux alentours de la cinquième semaine de grossesse et disparaissent à la fin du premier trimestre. Lorsque ces symptômes se prolongent au-delà du troisième mois, une consultation médicale est nécessaire. En cas de vomissements importants, il convient d'orienter la femme enceinte vers son médecin qui devra s'assurer de l'absence de tout risque de déshydratation ou de carences nutritionnelles, pour le fœtus comme pour la mère. Ces conseils restent valables en cas de difficultés à s'alimenter ou à boire suffisamment et si les vomissements induisent une perte de poids.

TEG1 Les aliments solides et riches en glucides sont à privilégier tandis que ceux riches en graisses et difficiles à digérer doivent être proscrits. Les repas peuvent être fractionnés pour éviter un apport trop important d'aliments. Si les nausées sont provoquées par les odeurs de nourriture, il est préférable de manger froid. 
TEG1 Peu de médicaments conseils peuvent être proposés aux patientes enceintes souffrant de ces symptômes. Les antihistaminiques tels que le dimenhydrinate (Mercalm ${ }^{\circledR}$, Nausicalm ${ }^{\circledR}$ ) sont contreindiqués tandis que les antagonistes dopaminergiques ne sont délivrés que sur prescription médicale (le métoclopramide peut entraîner des troubles neurologiques à type de syndromes extrapyramidaux qu'il conviendra d'identifier au plus vite en cas de symptômes caractérisés).

TEG1 Certaines plantes peuvent être conseillées, notamment les rhizomes de gingembre en infusion, l'écorce de citron ou les feuilles de menthe, en infusion. Quant aux huiles essentielles, elles ne doivent pas être utilisées durant le premier trimestre de la grossesse et le seront avec précaution à compter du quatrième mois.

TEG1 Les souches homéopathiques Sepia, Ignatia ou Nux vomica, en $7 \mathrm{CH}$ ou $9 \mathrm{CH}$, ne font pas courir de risque majeur à la mère et son fœtus.

\section{T1 La constipation}

TEG1 La constipation affecte plus de $\mathbf{8 0} \%$ des femmes enceintes. Elle peut survenir à tout moment de la grossesse etse définit comme une difficulté à évacuer les selles, dont la fréquence diminue (mois de trois par semaine).

TEG1 Une altération du mouvement péristaltique de l'intestin explique le plus souvent la survenue de la constipation mais celle-ci peut également être d'origine hormonale, secondaire à une alimentation pauvre en fibres ou une hydratation insuffisante ou encore être en lien avec certains facteurs psychologiques comme le stress [1]. Une consultation médicale s'impose en cas de chronicité, de présence de sang dans les selles, de fortes douleurs abdominales, de fièvre ou d'impossibilité à émettre des gaz, associée à des ballonnements et/ou des vomissements.

TEG1 La stratégie de prise en charge repose sur le respect de règles hygiéno-diététiques avant toute chose : avoir une alimentation variée et équilibrée riche en fibres alimentaires, boire 1,5 à 2 litres d'eau par jour et pratiquer un exercice physique adapté et régulier.

TEG1 Les laxatifs irritants tels que les anthracéniques (séné, bourdaine...) ou le bisacodyl (Contalax ${ }^{\circledR}$, Dulcolax ${ }^{\circledR}$ ) sont déconseillés durant la grossesse. Les laxatifs lubrifiants (Lubentyl ${ }^{\circledR}$, Lansoy ${ }^{\circledR}$ ) peuvent être utilisés sur une courte période afin d'éviter un risque de malabsorption des vitamines liposolubles. Enfin, les laxatifs osmotiques à base de lactulose (Duphalac ${ }^{\circledR}$, Transulose ${ }^{\circledR}$ ) doivent être préférés au macrogol (Forlax ${ }^{\circledR}$, Transipeg ${ }^{\circledR}$ ).

TEG1 Les seules plantes dont l'usage est reconnu dans cette indication chez la femme enceinte sont le Psyllium et l'Ispaghul. Les graines de ces deux plantes doivent être administrées par voie orale, avec un verre d'eau ou dans un yaourt.

Cle_illus1

T1 Les troubles veineux 
TEG1 Les troubles circulatoires et l'insuffisance veineuse apparaissent souvent dès le début de la grossesse et peuvent se présenter sous forme de sensations de lourdeur dans les jambes, de varices ou d'hémorroödes [4]. Les varices (30 à $50 \%$ des femmes enceintes) apparaissent à la faveur de diverses modifications physiologiques : diminution de la contractilité des veines, compression de la veine cave inférieure par l'utérus et modifications hormonales. Toutefois, d'autres causes sont identifiées telles que la sédentarité, la chaleur, le manque d'exercice, l'excès de poids ou les facteurs génétiques. Les hémorroïdes, secondaires à une dilatation anormale des veines de la muqueuse anale ou rectale, se caractérisent par des douleurs, des suintements, un prurit et des saignements.

TEG1 Avant la mise en place d'un traitement, quelques règles hygiéno-diététiques doivent être rappelées. Ainsi, la station debout prolongée doit être proscrite. Par ailleurs, il convient d'encourager une activité physique raisonnable que la femme enceinte peut faire suivre d'un massage des jambes sous l'eau froide. Les bains chauds ainsi que les expositions au soleil, qui provoquent une vasodilatation, doivent être évités.

TEG1 Les phlébotoniques sont recommandés en cas de troubles fonctionnels non compliqués : la diosmine (Dio ${ }^{\circledR}$, Diovenor ${ }^{\circledR}$, Mediveine $^{\circledR}$ ), la troxérutine (Veinamitol ${ }^{\circledR}$, Rheoflux ${ }^{\circledR}$ ) ou certains flavonoïdes comme l'hespéridine (Cyclo $3 \mathrm{Fort}^{\circledR}{ }^{\circledR}$ ) pour lutter contre les jambes lourdes et les troubles veineux. Ces traitements peuvent être complétés par une compression, sur prescription médicale : bandes pour un usage à court terme ; bas, chaussettes ou collants dans le cadre d'une utilisation à plus longue échéance.

TEG1 L'utilisation de Petit houx et de Vigne rouge est autorisée chez la femme enceinte : le rhizome de Petit houx sous forme d'extraits secs afin d'éviter le risque de colites lymphocytaires décrit avec les décoctions de cette plante ; les feuilles de Vigne rouge en infusions, consommées durant la journée.

\section{T1 Les troubles du sommeil}

TEG1 Les troubles du sommeil font partie des symptômes, bénins, fréquemment rapportés au comptoir par les femmes enceintes. Durant le premier trimestre de la grossesse, la fatigue s'accroît en raison de l'imprégnation hormonale, entraînant une envie irrépressible de dormir. Au cours du second semestre, cet accès de somnolence disparaît. Enfin, durant le dernier trimestre, les troubles du sommeil, à type d'insomnies, sont plus fréquents. Ils peuvent être dus à une anxiété grandissante à l'approche de l'accouchement, mais aussi à la difficulté de trouver une position confortable au lit ainsi qu'aux mouvements du bébé.

TEG1 Quelques règles assez simples peuvent être conseillées : éviter la consommation d'excitants et toute stimulation intellectuelle en soirée ; se coucher, dans la mesure du possible, chaque jour à la même heure ; veiller à la qualité de la literie (matelas ferme, oreiller plat et couvertures pas trop serrées).

TEG1 Parmi les molécules disponibles sur le marché, la doxylamine (Donormyl ${ }^{\circledR}$ ) peut être proposée chez la femme enceinte. Une surveillance doit bien évidemment être associée à cette délivrance, en particulier au cours du dernier trimestre de la grossesse durant lequel le risque de syndrome 
anticholinergique du nouveau-né est important. D’autres médicaments, exclusivement sur prescription médicale, peuvent être délivrés aux patientes dont les symptômes sont trop pénibles.

TEG1 Dans ce cadre, aucune plante ne peut être utilisée sans faire courir de risque. Le conseil officinal doit donc se limiter à la prescription de souches homéopathiques telles que Coffea cruda en cas d'insomnie liée à une suractivité avant le coucher et Ignatia si les insomnies sont provoquées par une inquiétude ou une anxiété.

TEG1 Des spécialités homéopathiques ou des formules composées sont utilisables par la femme enceinte : Passiflora composé ${ }^{\circledR}$, Homéogène ${ }^{\circledR} 46$, Sédatif $P C^{\circledR}$.

Cle_illus2

\section{T1 Les troubles ORL}

La prise en charge d'une rhinite ou d'une toux pendant la grossesse, en dehors de la fièvre, repose avant tout sur des mesures non pharmacologiques [5].

\section{T2 La rhinite}

TEG1 La prise en charge d'une rhinite sous sa forme allergique ne doit absolument pas reposer sur la prise d'antihistaminiques $\mathrm{H}_{1}$ dont les conséquences tératogènes ont été décrites chez l'animal. De même, les décongestionnants, tels que la phényléphrine ou la pseudo-éphédrine, sont contreindiqués durant la grossesse.

TEG1 En revanche, un traitement local reposant sur des solutions de lavage du nez (Prorhinel ${ }^{\circledR}$, Stérimar ${ }^{\circledast}$ ) associé à des spécialités homéopathiques $\left(\operatorname{Coryzalia}^{\circledR}\right.$, Sinuspax $\left.{ }^{\circledR}\right)$ doit être recommandé.

TEG1 Si les symptômes perdurent plus de huit jours, mais aussi en cas de fièvre, de douleur nasale ou sinusale avec un écoulement purulent important, il convient d'orienter la patiente vers son médecin traitant.

\section{Points à retenir}

- Les maux de la grossesse sont multiples et parfois invalidants.

- La plus grande vigilance doit être envisagée lors de la délivrance de médicament à une femme enceinte.

- Le respect de règles hygiéno-diététiques simples peut améliorer les maux au cours de la grossesse.

- Les antagonistes dopaminergiques sont proscrits chez la femme enceinte souffrant de nausées et vomissements. 
- L'usage de plantes et d'huiles essentielles doit être limité chez la femme enceinte en raison d'un risque iatrogène important.

\section{T2 La toux grasse et sèche}

TEG1 En cas de toux grasse, l'utilisation des mucolytiques, mucorégulateurs et des spécialités à base d'huiles essentielles appliquées sur la peau est proscrite durant la grossesse. Quelques souches homéopathiques peuvent être proposées comme Mercurius solubilis (toux grasse aggravée la nuit), Ipeca (toux grasse spasmodique importante).

TEG1 S'agissant des sirops utilisés dans le traitement de la toux sèche, ils sont déconseillés chez la femme enceinte du fait de l'absence de données suffisantes. Seule la spécialité Hélicidine ${ }^{\circledR}$ peut leur être proposée.

TEG1 La persistance de ces symptômes, accompagnés de fièvre, durant plus de cinq jours doit impérativement amener les patientes à consulter leur médecin traitant.

\section{T1 Conclusion}

Le rôle du pharmacien d'officine rappellera avant toute chose les règles hygiéno-diététiques à suivre pour lutter contre ces désagréments et ne manquera pas d'orienter la patiente vers son médecin traitant en cas d'aggravation des symptômes.

\section{Références}

[1] Battu C. Troubles digestifs et grossesse. Act Pharm. 2015;54(550):19-22.

[2] Lansac J, Magnin G, Sentilhes L. Obstétrique pour le praticien. Issy-les-Moulineaux: Elsevier Masson; 2013.

[3] Chassagnol-Clausade P. Les nausées et les vomissements. Act Pharm. 2008;47(477):8-12

[4] Battu C. Accompagnement d'une femme enceinte présentant des troubles circulatoires. Act Pharm. 2015;54(550):23-26

[5] Battu C. Les affections ORL chez la femme enceinte. Act Pharm. 2015;54(550):27-9.

Déclaration de liens d'intérêts

L'auteur déclare ne pas avoir de liens d'intérêts.

\section{Illustrations}




\section{Cle_illus1}

(C) ArtFamily/stock.adobe.com

Les troubles circulatoires et l'insuffisance veineuse apparaissent souvent dès le début de la grossesse et peuvent se présenter sous forme de sensations de lourdeur dans les jambes, de varices ou d'hémorroïdes.

Sur 1 colonne

\section{Cle_illus2}

\section{(C) Africa Studio/stock.adobe.com}

Durant le dernier trimestre de la grossesse, les troubles du sommeil sont plus fréquents, dus à une anxiété grandissante à l'approche de l'accouchement, à la difficulté de trouver une position confortable au lit ou aux mouvements nocturnes du bébé.

\section{Sur 1 colonne}



